\title{
Variabilidade espacial de propriedades dendrométricas do eucalipto e de atributos físicos de um Latossolo Vermelho
}

\author{
Gilberto Rosa Filho ('); Morel de Passos e Carvalho (1,*); Rafael Montanari (2); Janete Motta da Silva ('); \\ Glécio Machado Siqueira ( $\left.{ }^{3}\right)$; Edmar Casarin Zambianco (') \\ (') Universidade Estadual Paulista "Julio de Mesquita Filho" (UNESP), Faculdade de Engenharia de Ilha Solteira (FEIS), Caixa Postal \\ 31, 15385-000 Itha Solteira (SP). \\ (2) Universidade Estadual de Mato Grosso do Sul (UEMS), Rodovia Aquidauana, Km 12, 79200-000 Aquidauana (MS). \\ (3) Universidade de Santiago de Compostela (USC), 27002, Lugo, Espanha. \\ (*) Autor correspondente: morel@agr.feis.unesp.br
}

Recebido: 10/mai./2010; Aceito: 10/mar./2011.

\section{Resumo}

Este trabalho teve como objetivo analisar a variabilidade espacial de atributos de eucalipto (Eucalyptus urophylla S.T. Blake) e atributos físicos de um Latossolo Vermelho distrófico em Selvíria (MS). A área de estudo possui cerca de 1,98 ha e as amostras de planta e solo foram coletadas em uma grade de amostragem contendo 122 pontos sendo: 84 pontos com espaçamento regular de $15 \mathrm{~m}$ e 38 pontos com espaçamento regular de $5 \mathrm{~m}$. Os atributos de planta e solo analisados foram: perímetro na altura do peito, altura da planta, volume de madeira, resistência do solo à penetração e o teor de água no solo. Os atributos das plantas de eucalipto apresentaram moderada variabilidade espacial, ao passo que os atributos do solo apresentaram variabilidade moderada $\left(R P_{3}\right)$ e forte $\left(R P_{1}\right)$. O modelo exponencial foi o que mais se adequou para os ajustes dos atributos de planta e solo estudados. A correlação linear simples mostrou haver baixa correlação entre os atributos de Eucalyptus urophylla e os atributos físicos do solo.

Palavras-chave: manejo do solo, agricultura de precisão, compactação do solo.

\section{Spatial variability of dendrometric properties of eucalyptus and physical attributes of a Rhodic Eutrudox}

\section{Abstract}

The objective of this work was to investigate the spatial variability of attributes of eucalyptus (Eucalyptus urophylla S.T. Blake) and physical attributes of a Rhodic Eutrudox at Selvíria (MS). The study area has about 1.98 ha and samples of plant and soil were collected in a sampling grid containing 122 points: 84 points with regular spacing of $15 \mathrm{~m}$ and 38 points with regular spacing of $5 \mathrm{~m}$. The following attributes of plant and soil were analyzed: perimeter at chest height, height of the plant, volume of wood, soil penetration resistance and soil moisture content. The attributes of the eucalyptus plants showed medium spatial variability, while the attributes of the soil showed medium and low variability. The exponential model was the most appropriate settings for the attributes of plant and soil studies. Simple linear correlation showed low correlation between the attributes of Eucalyptus urophylla and the soil physical attributes.

Key words: soil management, precision farming, soil compaction. 


\section{INTRODUÇÃO}

Originário da Austrália, o Eucalyptus spp. foi introduzido no Brasil em 1904, sendo atualmente uma das essências florestais que mais tem atendido as necessidades de reposição de matéria-prima no processo industrial, principalmente no processo industrial de papel e celulose (Dossa et al., 2002; Ipef, 2009; Lima et al., 2010). Nos dias atuais, as plantaçôes de eucalipto ocupam, no Brasil, uma área de aproximadamente 3,5 milhôes de hectares, sendo produzidos comercialmente o volume de até $45 \mathrm{~m}^{3} \mathrm{ha}^{-1}$ ano $^{-1}$ de madeira, a partir de clones híbridos das espécies mais cultivadas (RAPAssi et al., 2008). O Eucalyptus urophylla (S.T. Blake) é uma planta de crescimento rápido, originária do Arquipélago de Sonda localizado no Nordeste da Austrália e se desenvolve desde o nível do mar até $1200 \mathrm{~m}$ de altitude, em temperaturas entre 24 e $28^{\circ} \mathrm{C}$ (Vieira e BuCsan, 1980). A espécie tem poucas exigências edáficas, o que a torna apropriada para reflorestamentos, tanto em solos inundados como em solos secos tropicais (NIETO e Rodriguez, 2003).

O estudo da relação entre a capacidade produtiva de povoamentos de Eucalyptus urophylla (S.T. Blake) e de atributos do solo favorece o manejo racional da floresta e do solo, evitando a degradação de seus atributos físicos e químicos, visando à produçáo sustentável (OrTIZ et al., 2006) e, sobretudo contribuindo para a aplicação de técnicas de silvicultura de precisão. Segundo Vettorazzi e Ferraz (2000), a silvicultura de precisão baseia-se na coleta e análise de dados geoespaciais, viabilizando intervençôes localizadas na floresta, com a exatidão e a precisão adequada.

Rigatto et al. (2004), descrevem que uma grande questáo a ser elucidada nos sistemas florestais é se as interferências na taxa de crescimento ocasionadas pelos atributos do solo podem proporcionar madeira de baixa qualidade. De acordo com Gonçalves et al. (1990) e RIGATTO et al. (2004) os atributos físicos do solo têm-se relacionado, com maior frequência, à capacidade produtiva dos sítios florestais e, conseqüentemente, com a qualidade da madeira produzida.

A qualidade física do solo para o desenvolvimento das plantas é determinada, dentre outros fatores, pela resistência do solo à penetração das raízes, sendo um atributo que merece destaque, já que atinge diretamente o desenvolvimento das plantas (PedrotTi et al., 2001). De acordo com Genro Junior et al. (2004) e Silva et al. (2004) a resistência do solo à penetração geralmente é influenciada pelas condiçóes do manejo e a intensidade de tráfego de máquinas agrícolas. DedeceK et al. (2005) estudando a produtividade de eucalipto em Neossolo, constataram que a melhor correlação do volume de madeira foi com a resistência à penetração, indicando a formação de camadas compactadas.

Theodorou et al. (1991) e Brady (1996) descrevem maiores taxas de crescimento nos sítios florestais, onde ocorrem menores valores de densidade do solo e a resistência à penetração de raízes e maiores valores de porosidade total, macroporosidade e disponibilidade de água. Lima et al. (2010), estudando a variabilidade espacial de atributos do solo e características dendrométricas de eucalipto, descrevem que a resistência do solo à penetração influenciou negativamente a produçáo de madeira.

De acordo com Rigatto et al. (2004), a taxa de crescimento do eucalipto em campo é diferenciada, justificando desta maneira o estudo detalhado de suas características dendrométricas e dos atributos do solo que interferem sobre o crescimento dos sistemas florestais. Nesse contexto, a aplicação de técnicas de geoestatística permite modelar e descrever a variabilidade espacial dos atributos de solo e planta, favorecendo a construção de mapas de isolinhas com o nível de detalhe desejável para maior compreensão das relaçóes entre o solo e as plantas.

O objetivo deste trabalho foi avaliar a variabilidade espacial de propriedades dendrométricas de Eucalyptus urophylla (S.T. Blake) e de atributos físicos de um Latossolo Vermelho distrófico em Selvíria (MS).

\section{MATERIAL E MÉTODOS}

O trabalho foi desenvolvido no ano agrícola de 2006/2007, no município de Selvíria (MS), na latitude $22^{\circ} 23^{\prime} \mathrm{S}$ e na longitude $51^{\circ} 27^{\prime} \mathrm{W}$, com precipitaçáo pluvial média anual de $1.300 \mathrm{~mm}$ e temperatura média de $23,7^{\circ} \mathrm{C}$. O tipo climático é o $\mathrm{A}_{\mathrm{w}}$, segundo a classificação de Köeppen, caracterizado como tropical úmido com estação chuvosa no verăo e seca no inverno. O solo, segundo o Sistema Brasileiro de Classificação de Solos (EMBrapa, 2006), é Latossolo Vermelho distrófico típico argiloso, A moderado, hipodistrófico, álico, caulinítico, mesoférrico, mesodênsico, mesocompactado, muito profundo, ácido.

As mudas de Eucalyptus urophylla (S.T. Blake) foram plantadas em 1992, obedecendo a um espaçamento regular de $3 \mathrm{~m} \times 3 \mathrm{~m}$. As amostragens das propriedades dendrométricas foram realizadas em uma grade amostral contendo 122 pontos, sendo 84 pontos com espaçamento de $15 \mathrm{~m} \mathrm{x} 15 \mathrm{~m}$ e 38 pontos com espaçamento de $5 \mathrm{~m} \times 5 \mathrm{~m}$, visando detalhar o estudo da dependência espacial (Figura 1).

As propriedades das plantas estudadas foram: perímetro na altura do peito (PAP) em $\mathrm{cm}$, altura das plantas (ALT) em m e volume de madeira (VOL) $\mathrm{em} \mathrm{m}^{3} \mathrm{ha}^{-1} \mathrm{ano}^{-1}$. $\mathrm{O}$ perímetro na altura do peito (PAP) foi medido a uma altura de aproximadamente $1,3 \mathrm{~m}$ utilizando fita métrica. A altura das árvores (ALT) foi medida utilizando dendrômetro. O volume total de madeira (VOL) foi determinado conforme descrito por CARVALHo (2000):

$V O L=\frac{\pi}{4}(D A P)^{2} * A L T^{*} F$ 


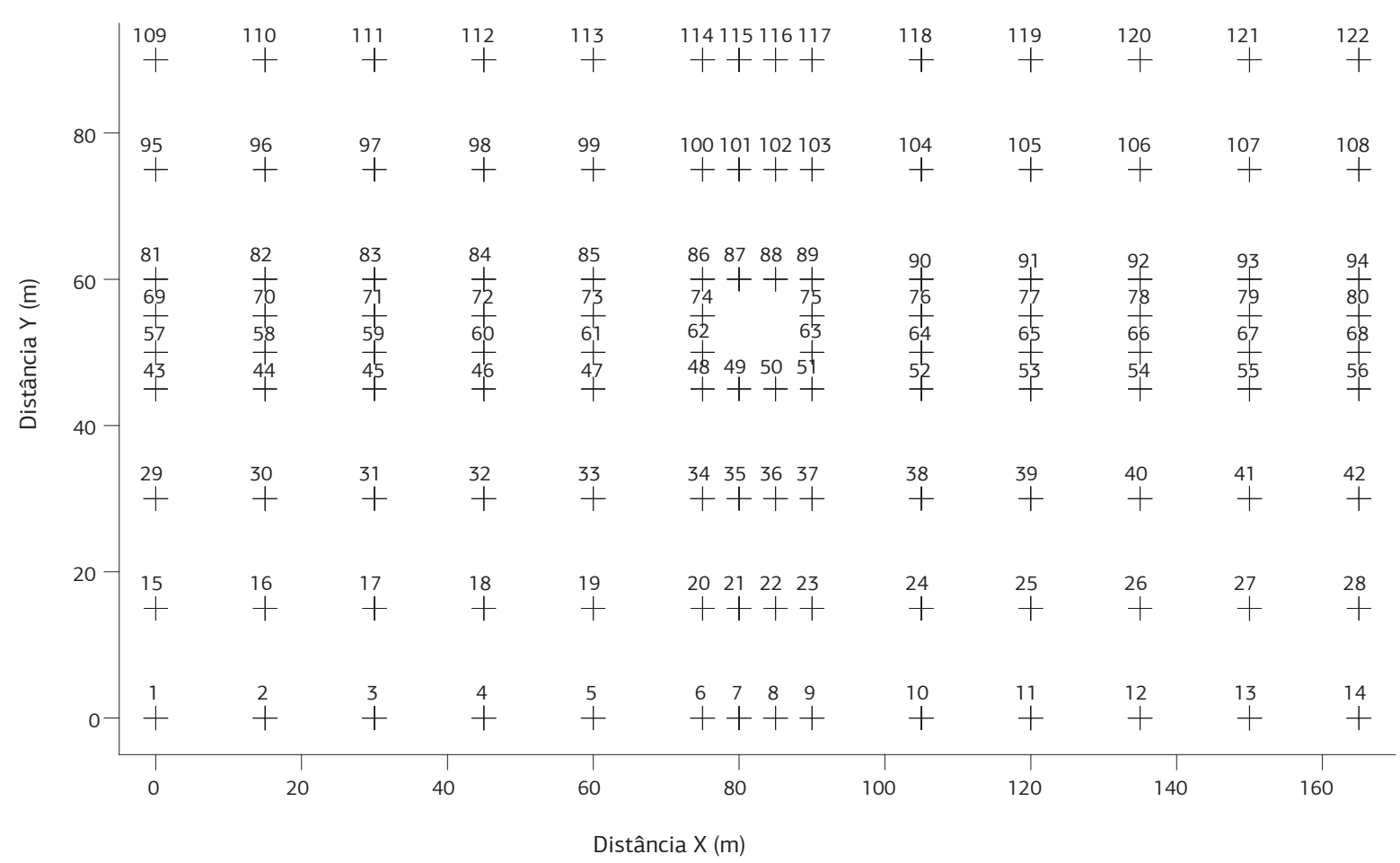

Figura 1. Esquema de coleta de amostras na área de estudo, contendo 122 pontos de amostragem.

VOL é o volume total de madeira $\left(\mathrm{m}^{3} \mathrm{ha}^{-1} \mathrm{ano}^{-1}\right)$; DAP é o diâmetro na altura do peito $(\mathrm{cm})$; ALT é a altura total das árvores (m) e $F$ é o fator de forma para as plantas de Eucalyptus urophylla (S.T. Blake).

$\mathrm{O}$ estudo dos atributos físicos foram realizados em agosto de 2006 sendo determinada a resistência do solo à penetração (RP) em MPa e o teor de água no solo (UG) em $\mathrm{kg} \mathrm{kg}^{-1}$, no entorno das plantas de eucalipto mais próximas ao ponto de amostragem. A resistência do solo a penetração foi medida até $0,30 \mathrm{~m}$ de profundidade utilizando um penetrômetro de impacto, seguindo as recomendaçóes de STOLF (1991), sendo posteriormente, estratificada em três camadas de estudo: 0,0-0,1 m (RP $\left.{ }_{1}\right)$, 0,1-0,2 $\mathrm{m}\left(\mathrm{RP}_{2}\right)$ e $0,2-0,3 \mathrm{~m}$ de profundidade $\left(\mathrm{RP}_{3}\right)$, estando o solo próximo à umidade na capacidade de campo. $\mathrm{O}$ teor de água no solo foi determinado nas três camadas de estratificação da resistência do solo à penetração (UG : 0,0-0,1 m; UG : 0,1-0,2 m e UG : 0,2-0,3 m), de acordo com metodologia descrita por EMBrApa (1997). Também foi determinada a resistência do solo à penetraçáo e o teor de água no solo média $\left(\mathrm{RP}_{\mathrm{M}}\right.$ e $\left.\mathrm{UG}_{\mathrm{M}}\right)$ para a profundidade de $0,0-0,3 \mathrm{~m}$.

Para cada atributo estudado foi efetuada a análise descritiva auxiliada pela estatística clássica. Utilizando-se o SAS (SChlotZhaver e Littel, 1997) foram calculados a média, mediana, moda, valores mínimo e máximo, desvio-padrão, coeficiente de variação, curtose e assimetria. A seguir, foram identificados os outliers (dados discrepantes), conforme identificação do gráfico de ramos e folhas, efetuando-se sua substituição pelo valor médio dos cir- cunvizinhos. Também foi efetuada a análise da distribuição de frequência por meio do teste de SHapiro e WiLK (1965) com 1\% probabilidade de erro.

A modelagem geoespacial foi realizada utilizando o programa GS 7.0 (RoberTson, 1998). Neste contexto, a análise inicial dos dados demonstrou que para alguns atributos a tendência, foi retirada, utilizando o método do refinamento da mediana destes atributos, conforme proposto por SiLva et al. (2003):

$N V=V O-(M L+M C)+M G$

NV representou o novo valor residual do atributo do qual foi retirada a tendência; $\mathrm{VO}$ o valor original do atributo do qual foi retirada a tendência; ML e MC representam, respectivamente, os valores da mediana da linha e da coluna nas quais estava o atributo; $M G$ representou a mediana geral dos atributos contidos na malha amostral.

Desta forma, pôde-se calcular novamente o semivariograma com os resíduos obtidos deste procedimento. $\mathrm{O}$ ajuste do semivariograma efetuado por meio da validação cruzada de acordo com ROBERTSON (1998) possibilitou a determinação dos parâmetros de ajuste do semivariograma experimental: efeito pepita $\left(\mathrm{C}_{0}\right)$, variância estrutural $\left(\mathrm{C}_{1}\right)$ e alcance (a). O grau de dependência espacial (GD) como também estudado por Montanari ET AL. (2010), onde valores $\leq 25 \%$ indicam grau de dependência espacial fraco, de $25 \%-75 \%$ indicam grau de dependência espacial moderado e $>75 \%$ indicam grau de dependência espacial forte entre amostras (Equação 3). 
$\mathrm{GD}=\left[\left(\frac{\mathrm{C}_{1}}{\mathrm{C}_{0}+\mathrm{C}_{1}}\right)\right] * 100$

A técnica de krigagem foi utilizada para estimar valores nos locais não amostrados por ser um estimador linear não enviesado. Uma vez determinados os valores para os locais não amostrados com a ajuda da técnica de krigagem, foram construídos mapas de isolinhas utilizando-se o programa GS+ (RoberTSON, I998), que emprega os mesmos valores estimados por meio da técnica de krigagem para determinação e localização das isolinhas; desta maneira, os mapas representam linhas bem definidas e embasadas em um algoritmo de regressão linear, conforme descrito por Carvalho e Assad (2005) e Siqueira et al. (2008).

\section{RESULTADOS E DISCUSSÃO}

Na tabela 1, observam-se parâmetros estatísticos dos atributos em estudo. De acordo com Gomes e Garcia (2002), a variabilidade de um atributo pode ser classificada por meio dos valores de coeficiente de variação $(\mathrm{CV})$ como baixo $(\leq 10 \%)$, médio $(10 \%-20 \%)$, alto $(20 \%-30 \%)$ e muito alto $(>30 \%)$. Verifica-se que dentre os atributos de Eucalyptus urophylla estudados apenas o volume de madeira (VOL) revelou coeficiente de variação muito alto $(36,5 \%)$, corroborando com o obtido por Lima et al. (2010) que estudaram o volume de madeira (VOL) para o Eucalyptus camaldulensis, constatando também coeficiente de variação muito alto $(30,3 \%)$. Nos demais atributos de Eucalyptus urophylla (PAP e ALT) os valores de coeficiente de variação foram medianos $(15,4 \%$ e 14,4\%), também concordando com Lima et al. (2010) que verificaram valores médios de coeficiente de variação para PAP e ALT de 11,8 e $12,0 \%$ respectivamente.

Conforme os resultados de Dossa et al. (2002), o valor médio observado neste estudo, para o volume de madeira (VOL $=64,3 \mathrm{~m}^{3} \mathrm{ha}^{-1} \mathrm{ano}^{-1}$ ), ficou bem acima da produtividade média brasileira de eucaliptos $\left(35 \mathrm{~m}^{3}\right.$ ha $^{-1}$ ano $^{-1}$ ) e muito próxima da produtividade de $60,0 \mathrm{~m}^{3}$ $\mathrm{ha}^{-1}$ ano $^{-1}$ para alguns clones de eucalipto das espécies mais plantadas no Brasil, como, por exemplo, o E. grandis e o E. saligna (Pereira et al., 2000). Contudo, os valores médios do PAP $(74,5 \mathrm{~cm})$ e do ALT $(31,0 \mathrm{~m})$ ficaram próximos daqueles verificados por Lima et al. (2010), que relataram para o Eucalyptus camaldulensis, em condições semelhantes às do presente estudo, valores do perímetro à altura do peito de $78 \mathrm{~cm}$ e da altura de $24 \mathrm{~m}$ (Tabela 1).

Os atributos físicos do solo $\mathrm{RP}_{1}$ e $\mathrm{UG}_{1}$ tiveram coeficiente de variação muito alto $(36,1 \%$ e $44,6 \%)$. A resistência do solo à penetração nas demais camadas de estudo $\left(\mathrm{RP}_{2}\right.$ e $\left.\mathrm{RP}_{3}\right)$ revelou altos valores de $\mathrm{CV}(26,3 \%$ $21,5 \%)$. O coeficiente de variação para $U_{2}$ e $U_{3}$ foi médio $(12,9 \%$ e $13,1 \%)$, discordando dos resultados analisados por Lima et al. (2010) que notaram coeficientes de variação médio para resistência a penetração e umidade, com valores entre $9,6 \%$ e $18,9 \%$

Verifica-se que os valores de C.V. para os atributos físicos do solo diminuem com o aumento da profundidade da camada em estudo. Todavia, esta tendência náo ocorre com os valores da média, uma vez que em $\mathrm{RP}_{3} \mathrm{o}$ maior valor foi médio $(4,870 \mathrm{MPa})$. A presença de maior valor de resistência do solo à penetração na profundidade de 0,2-0,3 m também é relatada por SANTOS et al. (2005) estudando a resistência do solo à penetração em Selvíria (MS), em Latossolo. A presença de maior valor de CV para $U_{1}(44,6 \%)$ pode ser justificada, considerando que no dia anterior à amostragem ocorreu chuva de $38 \mathrm{~mm}$ que pode ter contribuído para aumentar a heterogeneidade natural deste atributo na área de estudo, principalmente na profundidade de $0,0-0,1 \mathrm{~m}$. Siqueira et al. (2008), estudando a variabilidade espacial de diferentes atributos físicos do solo na mesma camada de estudo e um Latossolo Vermelho eutroférrico em Campinas (SP), descreve que a umidade na camada superficial do solo é de grande

Tabela 1. Parâmetros estatísticos para os atributos estudados

\begin{tabular}{|lcccccccccc} 
Atributo & Média & Mínimo & Máximo & DP & CV & Assimetria & Curtose & Pr<w & DF \\
\hline PAP $(\mathrm{cm})$ & 74,509 & 44,350 & 99,900 & 11,469 & 15,4 & $-0,125$ & $-0,325$ & 0,558 & $\mathrm{NO}$ \\
\hline $\mathrm{ALT}(\mathrm{m})$ & 31,062 & 20,400 & 41,000 & 4,471 & 14,4 & $-0,116$ & $-0,235$ & 0,541 & $\mathrm{NO}$ \\
\hline $\mathrm{VOL}\left(\mathrm{m}^{3} \mathrm{ha}^{-1} \mathrm{ano}^{-1}\right)$ & 64,296 & 17,463 & 122,720 & 23,464 & 36,5 & 0,297 & $-0,473$ & 0,143 & $\mathrm{NO}$ \\
\hline $\mathrm{RP}_{1}(\mathrm{MPa})$ & 2,242 & 1,030 & 4,037 & 0,127 & 36,1 & $-0,264$ & $-0,062$ & 0,237 & $\mathrm{LN}$ \\
\hline $\mathrm{RP}_{2}(\mathrm{MPa})$ & 3,893 & 1,416 & 8,241 & 0,155 & 26,3 & $-0,058$ & 0,051 & 0,032 & $\mathrm{TL}$ \\
$\mathrm{RP}_{3}(\mathrm{MPa})$ & 4,870 & 1,807 & 3,467 & 0,148 & 21,5 & $-0,155$ & $-0,376$ & 0,208 & $\mathrm{LN}$ \\
\hline $\mathrm{RP}_{\mathrm{M}}(\mathrm{MPa})$ & 2,055 & 0,793 & 4,325 & 0,136 & 10,4 & $-0,132$ & $-0,144$ & 0,566 & $\mathrm{LN}$ \\
\hline $\mathrm{UG}_{1}\left(\mathrm{~kg} \mathrm{~kg}{ }^{-1}\right)$ & 0,133 & 0,102 & 0,181 & 0,055 & 44,6 & 0,370 & $-0,316$ & 0,074 & $\mathrm{LN}$ \\
\hline $\mathrm{UG}_{2}\left(\mathrm{~kg} \mathrm{~kg}^{-1}\right)$ & 0,139 & 0,105 & 0,187 & 0,018 & 12,9 & 0,221 & 0,005 & 0,039 & $\mathrm{TN}$ \\
\hline $\mathrm{UG}_{3}\left(\mathrm{~kg} \mathrm{~kg}^{-1}\right)$ & 0,138 & 0,074 & 0,193 & 0,018 & 13,1 & $-0,036$ & 1,288 & 0,247 & $\mathrm{NO}$ \\
\hline $\mathrm{UG}_{\mathrm{M}}\left(\mathrm{kg} \mathrm{kg}^{-1}\right)$ & 0,139 & 0,087 & 0,216 & 0,027 & 19,2 & 0,115 & 0,022 & 0,087 & $\mathrm{NO}$ \\
\hline
\end{tabular}

DP: desvio-padrăo; C.V.: coeficiente de variaçấo (\%); DF: distribuiçăo de frequência com 1\% de probabilidade de erro por meio do teste de normalidade de SHAPIRO e WILK (1965). 
instabilidade, uma vez que pequenas variaçóes no solo e na atmosfera podem interferir nos resultados.

De acordo com Carvalho et al. (2002a), valores de assimetria e curtose próximos de zero, indicam que os dados revelam distribuição de frequência normal. Nesse contexto, verifica-se que PAP, ALT e VOL, $\mathrm{UG}_{3}$ e $\mathrm{UG}_{\mathrm{M}}$ indicam distribuição de frequência do tipo normal, coincidindo com outros estudos (Souza et al., 2001; CARVAlHo et al., 2002b; Dossa et al., 2002; SANTOS et al., 2005; Veronese Junior et al., 2006). Já em $\mathrm{RP}_{1}, \mathrm{RP}_{3}, \mathrm{RP}_{\mathrm{M}}$ e $\mathrm{UG}_{1}$ a distribuição de frequência é do tipo lognormal, enquanto a da $\mathrm{RP}_{2}$ tendeu a lognormal e a $\mathrm{UG}_{2}$ tendeu a normal.

Os valores de média para os atributos de Eucalyptus urophylla estão próximos dos valores determinados por Aguiar et al. (1995), estudando Eucalyptus citriodora aos 14 anos em Pradópolis (SP) e por Bila e Issufo (1994), estudando povoamentos florestais com Eucalyptus camaldulensis aos 10 anos em Moçambique. De acordo com a classificaçáo da resistência do solo à penetração, estabelecida por ARSHAD et al. (1996), os valores médios observados enquadraram-se na faixa de alta resistência do solo à penetração, com exceção do $\mathrm{RP}_{3}$ que foi muito alta. Estes resultados estáo de acordo com os de Lima et al. (2010) que encontraram valores altos de resistência à penetraçáo (6,4 a 9,6 MPa). Os valores de umidade aumentaram de 0,133 para $0,139 \mathrm{~kg} \mathrm{~kg}^{-1}$, mantendo-se constantes nas duas últimas (0,1-0,2 $\mathrm{m}$ e 0,2-0,3 $\mathrm{m}$ de profundidade).

$\mathrm{Na}$ tabela 2, observa-se a matriz de correlação linear simples entre os atributos de solo e planta. A correlação entre ALT x PAP $\left(r=0,677^{* *}\right)$ é elevada, seguindo o critério de classificação das amplitudes de $\operatorname{SHARP}(\mathrm{s} / \mathrm{d})$.

Para os atributos físicos do solo (RP e UG), verificase que a melhor correlação é descrita entre $U_{2} \times U_{M}$ $\left(r=0,974^{* *}\right)$. Observa-se que as correlaçóes entre os atributos do solo (RP e UG), foram negativas, em todas as camadas estudadas, corroborando com o observado por Theodorou et al. (1991), Brady (1996), Tormena et al. (1998) e Lima et al. (2010).
Entre os atributos de planta (ALT, PAP e VOL) e do solo (RP e UG), há de se destacar os valores do coeficiente de correlação entre PAP $\times \mathrm{RP}_{3}\left(r=-0,158^{\text {ns }}\right)$ e $\operatorname{VOL} \times \mathrm{RP}_{3}\left(r=-0,176^{\mathrm{ns}}\right)$, que mesmo sendo náo significativos indicando relação inversa entre as variáveis, ilustram que quanto menor a resistência à penetração maior será o volume de madeira, dando assim uma resposta de como manejar a área em estudo. Desta forma, esse fato está de acordo com Bellote e Dedecek (2006), que observaram a mesma tendência que com o aumento da resistência à penetração ocorra uma diminuição na produtividade, quando correlacionada com a produtividade de pinus em diferentes tipos de solos e com crescimentos variados.

Os parâmetros de ajuste do semivariograma experimental são apresentados na tabela 3 . O modelo esférico se ajustou apenas para os semivariogramas do perímetro na altura do peito (PAP), discordando de outros autores que descrevem este modelo como o que mais se ajusta aos atributos de solo e planta (Souza et al., 2001; Siqueira et al., 2008). Os semivariogramas da altura das árvores (ALT), do volume de madeira (VOL), e da resistência do solo à penetração de $0,0-0,1 \mathrm{~m}$ de profundidade $\left(\mathrm{RP}_{1}\right)$ e de 0,2-0,3 m de profundidade $\left(\mathrm{RP}_{3}\right)$ se ajustaram ao modelo exponencial. Os demais atributos tiveram efeito pepita puro, visto que o espaçamento utilizado náo foi suficiente para detectar a variabilidade espacial entre amostras.

De acordo com Siqueira et al. (2008), o efeito pepita $\left(\mathrm{C}_{0}\right)$ indica a descontinuidade espacial de um atributo e, portanto, quanto maior o valor $\mathrm{de}_{0}$, maior a variabilidade náo detectada durante o processo de amostragem. Nesse contexto, verifica-se que os valores de $\mathrm{C}_{0}$ (Tabela 3 ) para os atributos comdependência espacial, verificada pelo semivariograma experimental, são baixos, indicando boa acurácia dos métodos de amostragem destes atributos de solo e planta (PAP, ALT, VOL, $\mathrm{RP}_{1}$ e $\mathrm{RP}_{3}$ ).

$\mathrm{O}$ grau de dependência espacial (GD) mostra que $\mathrm{RP}_{1}$ tem forte dependência entre as amostras, enquanto nos demais atributos (PAP, ALT, VOL e RP 3 ), há moderada dependência

Tabela 2. Matriz de correlação linear para os atributos estudados

\begin{tabular}{lccccccccccc} 
& PAP & ALT & VOL & RP1 & RP2 & RP3 & RPM & UG1 & UG2 & UG3 & UGM \\
ALT & $0,677^{* *}$ & - & - & - & - & - & - & - & - & - \\
\hline VOL & $*$ & $*$ & - & - & - & - & - & - & - & - \\
\hline RP1 & 0,084 & $-0,112$ & 0,044 & - & - & - & - & - & - & - & - \\
\hline RP2 & $-0,013$ & 0,051 & 0,018 & $0,392^{* *}$ & - & - & - & - & - & - & - \\
\hline RP3 & $-0,158$ & $-0,076$ & $-0,176$ & 0,115 & $0,363^{* *}$ & - & - & - & - & - & - \\
\hline RPM & 0,013 & 0,072 & 0,037 & $0,371^{* *}$ & $0,966^{* *}$ & $0,265^{* *}$ & - & - & - & - & - \\
\hline UG1 & 0,126 & $-0,053$ & 0,065 & $-0,038$ & $-0,146$ & $-0,174$ & $-0,148$ & - & - & - & - \\
\hline UG2 & 0,127 & 0,018 & 0,073 & $-0,029$ & $-0,131$ & $-0,192$ & $-0,129$ & 0,157 & - & - & - \\
\hline UG3 & $-0,022$ & $-0,053$ & $-0,023$ & 0,057 & $-0,206^{*}$ & $-0,332^{* *}$ & $-0,181^{*}$ & 0,157 & $0,217^{*}$ & - \\
\hline UGM & 0,104 & 0,004 & 0,062 & $-0,016$ & $-0,157$ & $-0,249^{* *}$ & $-0,153$ & 0,174 & $0,974^{* *}$ & $0,419^{* *}$ & -
\end{tabular}

* A correlaçáo linear entre estes atributos não foi calculada uma vez que o perímetro na altura do peito (PAP) e a altura (ALT) săo utilizados no calculo do volume total de madeira (VOL). 
Tabela 3. Parâmetros do semivariograma para os atributos estudados

\begin{tabular}{|c|c|c|c|c|c|}
\hline Atributo & Modelo & $\mathrm{C}_{0}$ & $\mathrm{C}_{0}+\mathrm{C}_{1}$ & A & GD \\
\hline PAP & Esférico & 0,0409 & 0,1070 & 24,0 & 62,1 \\
\hline ALT & Exponencial & 0,4900 & 1,6200 & 28,5 & 69,6 \\
\hline VOL & Exponencial & 0,0123 & 0,0497 & 46,5 & 75,3 \\
\hline $\mathrm{RP}_{1}$ & Exponencial & 0,0092 & 0,0159 & 84,0 & 42,1 \\
\hline $\mathrm{RP}_{2}$ & Efeito pepita puro & & & & \\
\hline $\mathrm{RP}_{3}$ & Exponencial & 0,0101 & 0,0205 & 60,6 & 50,2 \\
\hline $\mathrm{RP}_{\mathrm{M}}$ & Efeito pepita puro & & & & \\
\hline $\mathrm{UG}_{1}$ & Efeito pepita puro & & & & \\
\hline $\mathrm{UG}_{2}$ & Efeito pepita puro & & & & \\
\hline $\mathrm{UG}_{3}$ & Efeito pepita puro & & & & \\
\hline$U G_{M}$ & Efeito pepita puro & & & & \\
\hline
\end{tabular}

a) PAP

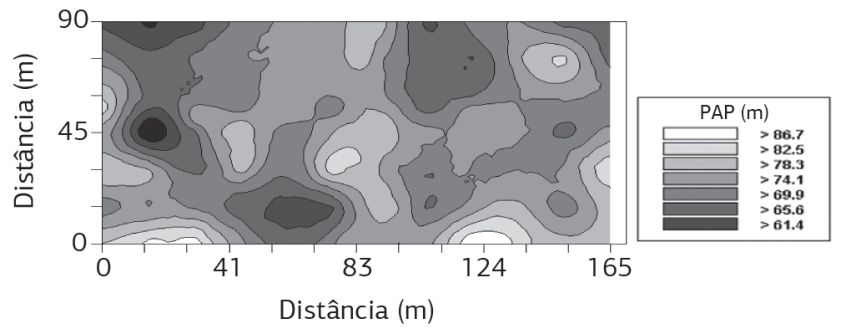

c) VOL

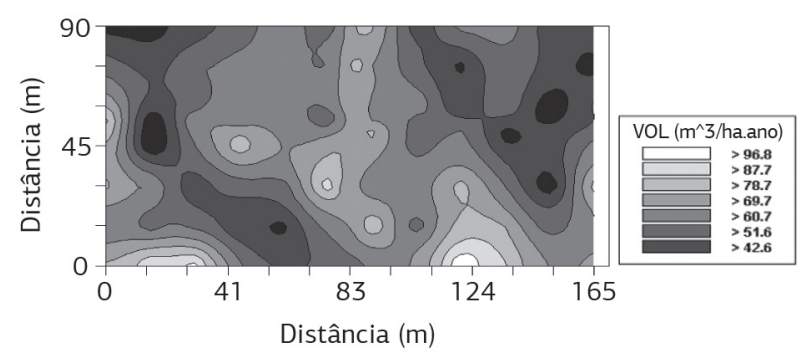

b) ALT

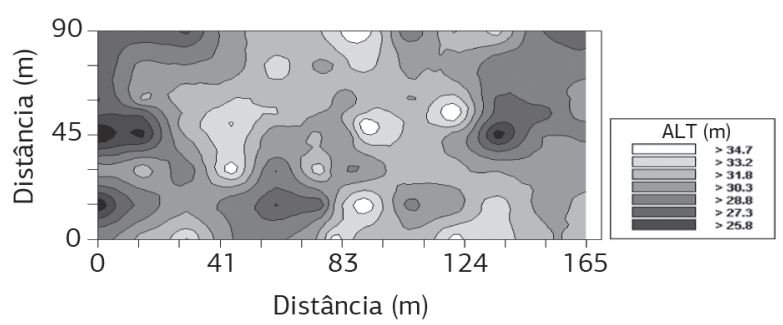

d) RP1

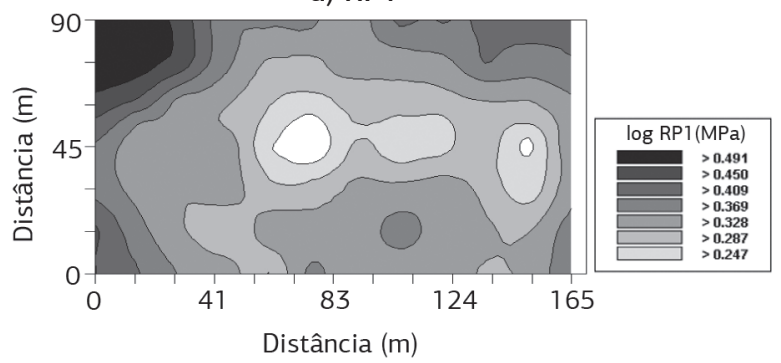

e) RP3

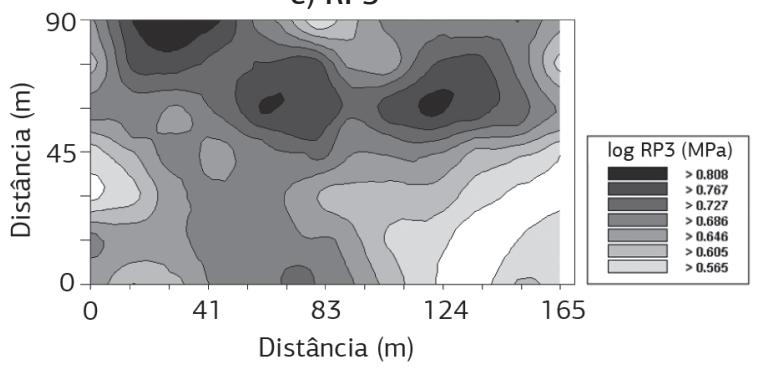

Figura 2. Mapas de variabilidade espacial de propriedades dendrométricas de Eucalyptus urophylla e de atributos físicos do solo em Selvíria (MS). Em (a): Perímetro a altura do peito; (b): Altura; (c): Volume de madeira; (d) e (e): Resistência à penetração de 0-0,1 e de 0,2-0,3m.

espacial de acordo com a classificação proposta por ZIMBACK (2001).

Com relação ao alcance (a) da dependência espacial, verifica-se que o perímetro na altura do peito (PAP), a altura das árvores (ALT) e o volume de madeira (VOL) respectivamente, foram de $24,0 \mathrm{~m}, 28,5 \mathrm{~m}$ e $46,5 \mathrm{~m}$. Por outro lado, a resistência do solo à penetraçáo $\left(\mathrm{RP}_{1}\right.$ e $\left.\mathrm{RP}_{3}\right)$ revela valores de alcance de $84,0 \mathrm{~m}$ e $60,6 \mathrm{~m}$ respectivamente. SAnTos et al. (2005) e Souza et al. (2006), estudando a va- riabilidade da resistência do solo à penetraçáo encontraram valores similares aos encontrados neste estudo, na mesma classe de solo e nas mesmas camadas de estudo.

Os mapas de variabilidade espacial (Figura 2) das propriedades dendrométricas de Eucalyptus urophylla (PAP, ALT e VOL) mostram que não há uma relação definida entre a variabilidade do perímetro na altura do peito (PAP) e a das demais propriedades de Eucalyptus urophylla estudadas (ALT e VOL). Este fato não era esperado uma vez 
que o PAP é calculado considerando a ALT e VOL. Todavia, quando comparados os mapas da altura das árvores (ALT) e do volume de madeira (VOL), verifica-se que há um determinado padrão na distribuiçáo espacial das linhas de contorno. Em ternos gerais, pode-se descrever que a diferenciação entre os mapas de variabilidade espacial dos atributos de Eucalyptus urophylla (PAP, ALT e VOL), envolvidos neste estudo, são consequência da interação das plantas com o ambiente. Com relaçáo aos atributos físicos do solo envolvidos neste estudo (RP e UG), pode-se descrever que não há alguma relação entre os mapas de variabilidade espacial, que possam justificar qualquer alteração no desenvolvimento das plantas de Eucalyptus urophylla.

A distribuição das linhas de contorno dos mapas de variabilidade espacial da resistência do solo à penetração (Figura 2) $\mathrm{RP}_{1}(0,0-0,1 \mathrm{~m})$ e $\mathrm{RP}_{3}(0,2-0,3 \mathrm{~m})$ mostra que seu padrão é distinto para as duas profundidades. Percebese que em ambos os mapas os maiores valores de resistência do solo à penetraçáo estáo localizados na parte superior esquerda da área de estudo.

A comparação entre os mapas de variabilidade espacial dos atributos de Eucalyptus urophylla e os atributos do solo revela que não há um padrão de similaridade. Conforme discutido anteriormente, os dados da correlação linear simples (Tabela 2) mostram que há uma função inversa entre PAP x $\mathrm{RP}_{3}(-0,158)$ e VOL $x \mathrm{RP}_{3}(-0,176)$, no entanto, nos mapas de variabilidade espacial destes atributos náo é possível determinar esta relaçáo de causa e efeito, devido à elevada variabilidade espacial destes parâmetros na área de estudo.

\section{CONCLUSÃO}

As correlaçôes lineares simples entre o volume de madeira do Eucalyptus urophylla e os atributos do solo foram significativas quando pareadas com a resistência à penetração apenas para a profundidade de 0,2 a 0,3 m. Os mapas de variabilidade espacial das características dendrométricas de Eucalyptus urophylla e da resistência do solo à penetração não demonstram nenhuma relação que possa explicar como o desenvolvimento das árvores na área de estudo é afetado pela resistência do solo na camada de $0,0-0,1 \mathrm{~m}$ e de $0,2-0,3 \mathrm{~m}$ de profundidade.

\section{AGRADECIMENTOS}

Os autores agradecem à Coordenação de Aperfeiçoamento de Pessoal de Nível Superior - CAPES, pela concessão de bolsa de estágio de doutoramento para Rafael Montanari na Universidad de la Coruña - UDC (BEX 5125/06-5). Ao Ministério de Assuntos Exteriores e de Cooperação do Governo da Espanha - MAEC-AECID, pela concessão de bolsa de doutorado para Glécio Machado Siqueira, na Universidade de Santiago de Compostela.

\section{REFERÊNCIAS}

AGUIAR, I.B.; VALERI, S.V.; SPINELLI, P.; SARTORI FILHO, A.; PIRES, C.A.M. Efeitos de intensidade de desbaste sobre o crescimento em altura e diâmetro de Eucalyptus citriodora Hook. IPEF, Piracicaba, n.48/49, p.1-7, 1995.

ARSHAD, M.A.; LOWERY, B.; GROSSMAN, B. Physical tests for monitoring soil quality. In: DORAN, J.W.; JONES, A.J. (Org.). Methods for assessing soil quality. Madison: Soil Science Society of America, 1996. p.123-141. (SSSA Special publication, 49)

BELLOTE, A.F.J.; DEDECEK, R.A. Atributos Físicos e Químicos do Solo e suas Relaçóes com o Crescimento e a Produtividade do Pinus taeda. Colombo: Embrapa Florestas, 2006. p.21-38. 2006. (Boletim de Pesquisa Florestal, 53)

BILA, A.; ISSUFO, A.A.K. Teste de procedências de Eucalyptus camaldulensis na regiáo Norte e Sul de Moçambique. IPEF, Piracicaba, n.47, p.50-55, 1994.

BRADY, N. C. The nature and properties of soils. New Jersey: Prentice Hall, 1996. 639p.

CARVALHO, A.M. Valorização da madeira do híbrido Eucaliptus grandis x Eucaliptus Urophilla através da produção conjunta de madeira serrada em pequenas dimensóes, celulose e lenha. Piracicaba, 2000. 129p. Tese (Mestrado) Escola Superior de Agricultura Luiz de Queiroz, Universidade de São Paulo, Piracicaba, 2000.

CARVALHO, J.R.; ASSAD, E.D. Análise espacial da precipitação pluviométrica no Estado de São Paulo: comparação de métodos de interpolação. Engenharia Agrícola, v.25, p.377-384, 2005.

CARVALHO, J.R.P.; SILVEIRA, P.M.; VIEIRA, S.R. Geoestatística na determinação da variabilidade espacial de características químicas do solo sob diferentes preparos. Pesquisa Agropecuária Brasileira, v.37, p.1151-1159, 2002a.

CARVALHO, M.P.; SORATTO, R.P.; FREDDI, O.S. Variabilidade espacial de atributos físicos em um Latossolo Vermelho Distrófico sob preparo convencional em Selvíria (MS). Acta Scientiarum Agronomy, v.24, p.1353-1361, 2002b.

DEDECEK, R.A.; GAVA, J.L. Influência da compactação do solo na produtividade da rebrota de eucalipto, Revista Árvore, v.29, p.383-390, 2005.

DOSSA, D.; SILVA, H.D.; BELLOTE, A.F.J.; RODIGHERI, H.R. Produção e rentabilidade do eucalipto em empresas florestais. Colombo: EMBRAPA, 2002. 4p.

EMPRESA BRASILEIRA DE PESQUISA AGROPECUÁRIA - EMBRAPA. Manual de métodos de análise de solos. Serviço Nacional de Levantamento e Conservação do Solo. 2.ed. Rio de Janeiro: EMBRAPA, 1997, 212p.

EMPRESA BRASILEIRA DE PESQUISA AGROPECUÁRIA - EMBRAPA. Sistema brasileiro de classificação de solos. Rio de Janeiro: EMBRAPA-CNP/Solos, 2006. 306p.

GENRO JUNIOR, S.A., REINERT, D.J.; REICHERT, J.M. Variabilidade temporal da resistência à penetração de um latossolo 
argiloso sob semeadura direta com rotaçáo de culturas. Revista Brasileira de Ciência do Solo, v.28, p.477-484, 2004.

GOMES, F.P., GARCIA, C.H. Estatística aplicada a experimentos agronômicos e florestais. Piracicaba: FEALQ, 2002. 309p.

GONÇALVES, J.L.M.; DEMATTÊ, J.L.I.; COUTO, H.T.Z. Relaçóes entre a produtividade de sítios florestais de Pinus taeda e Pinus elliottii com as propriedades de alguns solos de textura arenosa e média no Estado de Sáo Paulo. Sáo Paulo: IPEF, 1990. p.24-39. (Boletim Técnico IPEF, n. 43/44)

INSTITUTO DE PESQUISA E ESTUDOS FLORESTAIS IPEF. Pesquisa florestal no Brasil: Tecnologia de produtos florestais. Disponível em: <http://www.ipef.br/mct/ MCT_04.htm>. Acesso em: 10 abr. 2009.

LIMA, C.G.R.; CARVALHO, M.P.; NARIMATSU, K.C.P.; SILVA, M.G.; QUEIROZ, H.A. Atributos físico-químicos de um latossolo vermelho do cerrado brasileiro e sua relaçáo com características dendrométricas do eucalipto. Revista Brasileira de Ciência do Solo, v.34, p.163-173, 2010

MONTANARI, R.; CARVALHO, M. P.; ANDREOTTI, M.; DLACHIAVON, F. C.; LOVERA, L. H.; HONORATO, M. A. O. Aspectos da produtividade do feijão correlacionados com atributos físicos do solo sob elevado nível tecnológico de manejo, Revista Brasileira de Ciência do Solo, Viçosa, v.34, p.1811-1822, 2010.

NIETO, V.M.; RODRIGUEZ, J. Eucalyptus urophylla Dehnh. In: VOZZO, J.A. Tropical Tree Seed Manual. Part II. Species Descriptions. Washington, D.C.: USDA Forest Service, 2003. p.473.

ORTIZ, J.L. ; VETTORAZZI, C.A. ; COUTO, H.T.Z. ; GONCALVES, J.L.M. . Relaçôes espaciais entre o potencial produtivo de um povoamento de eucalipto e atributos do solo e do relevo. Scientia Forestalis, v.72, p.67-79, 2006.

PEDROTTI, A.; PAULETTO, E.A.; CRESTANA, S.; FERREIRA, M.M.; DIAS JUNIOR., M.S.; GOMES, A.S.; TURATTI, A.L. Resistência mecânica à penetração de um Planossolo submetido a diferentes sistemas de cultivo. Revista Brasileira de Ciência do Solo, v.25, p.521-529, 2001.

PEREIRA, J.C.D.; STURION, J.A.; HIGA, A.R.; HIGA, R.C.V.; SHIMIZU, J.Y. Características da madeira de algumas espécies de eucalipto plantadas no Brasil. Colombo: Embrapa Florestas, 2000. 113p. (Embrapa Documentos, 38)

RAPASSI, R.M.A.; TARSITANO, M.A.A.; PEREIRA, J.C.R.; ARAÚJO, C.A.M. Cultura do eucalipto na região de Suzanápolis, Estado de Sáo Paulo: Análise econômica. Informaçóes Econômicas, v.38, p.7-13, 2008.

RIGATTO, P.A.; DEDECEK, R.A.; MATOS, J.L.M. Influência dos atributos do solo sobre a qualidade da madeira de Pinus taeda para produção de celulose Kraft. Revista Árvore, v.28, p.267.273, 2004.

ROBERTSON, G.P. GS+: Geostatistics for the environmental sciences. Michigan: Gamma Desing Software, 1998. 151p.

SANTOS, P.A.; CARVALHO, M.P.; FREDDI, O.S.; KITAMURA, A.E.; FREITAG, E.E.; VANZELA, L.S. Correlação linear e espacial entre o rendimento de grãos do feijoeiro e a resistência mecânica à penetração em um Latossolo Vermelho Distrófico, Revista Brasileira de Ciência do Solo, v.29, p.287-295, 2005.

SCHLOTZHAVER, S.D.; LITTELL, R.C. SAS system for elementary statical analysis. 2.ed. Cary: SAS, 1997. 441p.

SHAPIRO, S.S.; WILK, M.B. An analysys of variance test for normality: complete samples. Biometrika, v.52, p.591-611, 1965.

SHARP. Instructor manual. Scientific computer. Model EL-5500 II. Japan: Sharp Eletronics Corporation, s/d.

SILVA, V.R.; REICHERDT, J.M.; STORCK, L.; FEIJÓ, S. Variabilidade espacial das características químicas do solo e produtividade de milho em um Latossolo Vermelho-Amarelo distrófico arênico. Revista Brasileira de Ciência do Solo, v.27, p.1013-1020, 2003.

SILVA, V.R.; REICHERT, J.M.; REINERT, D.J. Variabilidade espacial da resistência do solo à penetração em plantio direto. Ciência Rural, v.34, p.399-406, 2004.

SIQUEIRA, G.M.; VIEIRA, S.R.; CEDDIA, M.B. Variabilidade espacial de atributos físicos do solo determinados por métodos diversos. Bragantia, v.67, p.203-211, 2008.

SOUZA, Z.M.; CAMPOS, M.C.C.; CAVALCANTE, I.H.L.; MARQUES, J.J.; CESARIN, L.G.; SOUZA, S.R. Dependência espacial da resistência do solo à penetraçáo e do teor de água do solo sob cultivo contínuo de cana-de-açúcar. Ciência Rural, v.36, p.128-134, 2006.

SOUZA, Z.M.; SILVA, M.L.S.; GUIMARÃES, G.L.; CAMPOS, D.T.S.; CARVALHO, M.P.; PEREIRA, G.T. Variabilidade espacial de atributos físicos em um Latossolo Vermelho Distroférrico sob semeadura direta em Selvíria (MS). Revista Brasileira de Ciência do Solo, v.25, p.699-707, 2001.

STOLF, R. Teoria e teste experimental de fórmulas de transformação dos dados de penetrômetro de impacto em resistência do solo. Revista Brasileira de Ciência do Solo, v.15, p.229-235, 1991.

THEODOROU, C.; CAMERON, J.N.; BOWEN, G.D. Growth of roots of differents Pinus radiata genotypes at different strength and aeration. Australian Forestry Resources, v.54, p.52-59, 1991.

TORMENA, C.A.; ROLOFF, G.; SÁ, J.C.M. Propriedades físicas do solo sob plantio direto influenciado por calagem, preparo inicial e tráfego. Revista Brasileira de Ciência do Solo, v.22, p.301-309, 1998.

VERONESE JUNIOR., V.; CARVALHO, M.P.; DAFONTE, J.; FREDDI, O.S.; VIDAL VÁZQUEZ, E.; INGARAMO, O.E. Spatial variability of soil water content and mechanical resistance of Brazilian ferralsol. Soil and Tillage Research, v.85, p.166-177, 2006.

VETTORAZZI, C.A; FERRAZ, S.F.B. Silvicultura de precisão: uma nova perspectiva para o gerenciamento de atividades florestais. In: BORÉM, A; GIUDICE, M.P.; MANTOVANI, E.C.; QUEIROZ, D.M.; FERREIRA, L.R.; VALLE, F.X.R.; GOMIDE, R.L. (Ed.). Agricultura de Precisão. Viçosa,: UFV, 2000. p.65-75.

VIEIRA, F.S.; BUCSAN, B. Ocorrência naturais de Eucalyptus urophylla na Indonésia. Silvicultura, p.359-361, 1980. 